Appl. Set-Valued Anal. Optim. 3 (2021), No. 2, pp. 133-147

Available online at http://asvao.biemdas.com

https://doi.org/10.23952/asvao.3.2021.2.01

\title{
GENERIC PROPERTIES OF OPERATOR-DEPENDENT NORMAL MAPPINGS
}

\author{
KAY BARSHAD, SIMEON REICH*, ALEXANDER ZASLAVSKI \\ Department of Mathematics, The Technion - Israel Institute of Technology, 32000 Haifa, Israel
}

\begin{abstract}
We introduce the notions of operator-dependent normality and operator-dependent weak normality with respect to a given convex function, which is uniformly continuous on bounded sets, and a given operator. In 2001, Gabour, Reich and Zaslavski considered bounded sets and studied the properties of normal mappings and normal sequences of mappings with respect to an everywhere uniformly continuous convex function. In 2020, Barshad, Reich and Zaslavski studied similar properties for not necessarily bounded sets, and also introduced the more general notion of weak normality. In this paper, we investigate the analogous properties regarding certain developments of these concepts and present some applications to the minimization of convex functions.

Keywords. Baire category; Banach space, Lyapunov function; Minimization problem; Normal mapping.
\end{abstract}

\section{INTRODUCTION AND BACKGROUND}

Let $(X,\|\cdot\|)$ be a normed space with norm $\|\cdot\|$. Let $K \subset X$ be a nonempty, closed and convex subset of $X$, and let $f: K \rightarrow \mathbb{R}$ be a convex function, which is bounded from below and uniformly continuous on bounded subsets of $K$. In the case where $K$ is an unbounded set, we also assume

$$
\lim _{x \rightarrow \infty} f(x)=\infty
$$

Set

$$
\inf f:=\inf \{f(x): x \in K\} \text {. }
$$

Denote by $\mathfrak{B}$ the set of all bounded mappings $B: K \rightarrow K$, by $\mathfrak{A}$ the set of all mappings $A \in \mathfrak{B}$, which satisfy

$$
f(A x) \leq f(x) \text { for each } x \in K
$$

and by $\mathfrak{A}_{c}$ the set of all continuous mappings $A \in \mathfrak{A}$. For the set $\mathfrak{A}$, we define a metric $\rho$ : $\mathfrak{A} \times \mathfrak{A} \rightarrow \mathbb{R}$ by

$$
\rho(A, B):=\sup \{\|A x-B x\|: x \in K\}, A, B \in \mathfrak{A} .
$$

Clearly, the metric space $\mathfrak{A}$ is complete if $(X,\|\cdot\|)$ is a Banach space, and the metric space $\mathfrak{A}_{c}$ is a closed subset of $\mathfrak{A}$. Denote by $\mathfrak{M}$ the set of all sequences of elements in $\mathfrak{A}$ and by $\mathfrak{M}_{c}$ the set

\footnotetext{
${ }^{*}$ Corresponding author.

E-mail addresses: kaybarshad@ technion.ac.il (K. Barshad), sreich@technion.ac.il (S. Reich), ajzasl@technion. ac.il (A. Zaslavski).

Received April 28, 2020; Accepted July 30, 2020.
}

(C)2021 Applied Set-Valued Analysis and Optimization 
of all sequences of elements in $\mathfrak{A}_{c}$. For the set $\mathfrak{M}$, we consider the following two uniformities and the topologies induced by them. The first uniformity is determined by the following basis:

$$
E_{1}(N, \varepsilon)=\left\{\left(\left\{A_{n}\right\}_{n=1}^{\infty},\left\{B_{n}\right\}_{n=1}^{\infty}\right) \in \mathfrak{M} \times \mathfrak{M}: \rho\left(A_{n}, B_{n}\right)<\varepsilon, n=1, \ldots, N\right\},
$$

where $N=1,2 \ldots$ and $\varepsilon>0$. This uniformity induces a uniform topology on $\mathfrak{M}$, which we denote by $\tau_{1}$ and call the weak topology.

The second uniformity is determined by the following basis:

$$
E_{2}(\varepsilon)=\left\{\left(\left\{A_{n}\right\}_{n=1}^{\infty},\left\{B_{n}\right\}_{n=1}^{\infty}\right) \in \mathfrak{M} \times \mathfrak{M}: \rho\left(A_{n}, B_{n}\right)<\varepsilon, n=1,2, \ldots\right\},
$$

where $\varepsilon>0$. This uniformity induces a uniform topology on $\mathfrak{M}$, which we denote by $\tau_{2}$ and call the strong topology. Clearly, $\tau_{2}$ is indeed stronger than $\tau_{1}$.

It is not difficult to see that the uniform spaces $\left(\mathfrak{M}, \tau_{1}\right)$ and $\left(\mathfrak{M}, \tau_{2}\right)$ are metrizable (by metrics $\rho_{1}$ and $\rho_{2}$, respectively) and complete if $(X,\|\cdot\|)$ is a Banach space. More information on uniformities and uniform spaces can be found, for example, in [1].

Clearly, $\mathfrak{M}_{c}$ is a closed subset of $\mathfrak{M}$ with respect to the weak topology (and therefore with respect to the strong topology) and hence complete with respect to both the strong and weak topologies. Denote by $\mathfrak{M}_{b}$ the set of all bounded sequences of elements in $\mathfrak{A}$ and by $\mathfrak{M}_{b c}$ the set of all bounded sequences of elements in $\mathfrak{A}_{c}$. It can easily be verified that $\mathfrak{M}_{b}$ and $\mathfrak{M}_{b c}$ are closed subsets of $\mathfrak{M}$ with respect to the strong topology. Evidently, the relative strong topology on $\mathfrak{M}_{b}$ is determined by the metric $d: \mathfrak{M}_{b} \times \mathfrak{M}_{b} \rightarrow \mathbb{R}$ defined by

$$
d\left(\left\{A_{n}\right\}_{n=1}^{\infty},\left\{B_{n}\right\}_{n=1}^{\infty}\right):=\sup \left\{\rho\left(A_{n}, B_{n}\right)\right\}_{n=1}^{\infty}\left\{A_{n}\right\}_{n=1}^{\infty},\left\{B_{n}\right\}_{n=1}^{\infty} \in \mathfrak{M}_{b} .
$$

For each $B_{0} \in \mathfrak{B}$, we set

$$
d_{B_{0}}:=\sup \left\{\left|f\left(B_{0} x\right)\right|: x \in K\right\} \text { and } S_{B_{0}}:=\left\{x \in K: f(x) \leq d_{B_{0}}\right\} .
$$

Note that the assumption that $f$ is convex and uniformly continuous on bounded sets implies that $d_{B_{0}}$ is finite. Note also that the set $S_{B_{0}}$ is always bounded (in the case where $K$ is unbounded this follows from (1.1)) and hence $f$ is uniformly continuous on it.

Definition 1.1. Let $B_{0} \in \mathfrak{B}$. A mapping $A: K \rightarrow K$ is said to be $B_{0}$-normal with respect to $f$ if for given $\varepsilon>0$, there is $\delta(\varepsilon)>0$ such that for each $x \in S_{B_{0}}$ satisfying $f(x) \geq \inf (f)+\varepsilon$, the inequality

$$
f(A x)<f(x)-\delta(\varepsilon)
$$

is true. A sequence $\left\{A_{n}\right\}_{n=1}^{\infty}$ of operators $A_{n}: K \rightarrow K$ is said to be $B_{0}$-normal with respect to $f$ if for given $\varepsilon>0$, there is $\delta(\varepsilon)>0$ such that for each $x \in S_{B_{0}}$ satisfying $f(x) \geq \inf (f)+\varepsilon$ and each integer $n=1,2, \ldots$, the inequality

$$
f\left(A_{n} x\right)<f(x)-\delta(\varepsilon)
$$

holds.

Example 1.1. Let $X=\mathbb{R}$ and $K=[0, \infty)$. Define $B_{0} \in \mathfrak{B}$ by $B_{0}(x):=|\cos x|$ for each $x \in K$,

and define $A: K \rightarrow K$ by $A x:=\left\{\begin{array}{ll}2^{-1} x, & x \leq 1, \\ 2 x-3 \cdot 2^{-1}, & 1<x \leq 3 \cdot 2^{-1}, \\ x, & 3 \cdot 2^{-1}<x \leq 2, \\ 2, & x>2,\end{array}\right.$ for each $x \in K$. Let $f: K \rightarrow \mathbb{R}$

be defined by $f(x):=x^{2}$ for each $x \in K$. Evidently, $f$ is convex and uniformly continuous 
on bounded sets. Clearly, $A$ is $B_{0}$-normal with respect to $f$. We also have $A \in \mathfrak{A}_{c}$, that is, $\mathfrak{A}_{c} \subset \mathfrak{A} \neq \emptyset$ and therefore $\mathfrak{M}_{c} \subset \mathfrak{M} \neq \emptyset$.

Definition 1.2. Let $B_{0} \in \mathfrak{B}$. A sequence $\left\{A_{n}\right\}_{n=1}^{\infty}$ of operators $A_{n}: K \rightarrow K$ is said to be $B_{0^{-}}$ weakly normal with respect to $f$ if for given $\varepsilon>0$, there exists a sequence $\left\{\delta_{n}\right\}_{n=1}^{\infty}$ of positive numbers such that $\limsup _{n \rightarrow \infty} n \delta_{n}=\infty$ and for each positive integer $n$, each $x \in S_{B_{0}}$ satisfying $f(x) \geq \inf (f)+\varepsilon$ and each integer $k=1,2, \ldots, n$, the inequality

$$
f\left(A_{k} x\right)<f(x)-\delta_{n}
$$

holds.

Remark 1.1. Assume $B_{0} \in \mathfrak{B}$. It is not difficult to see that for each $\alpha \in(0,1)$ and each $\left\{A_{n}\right\}_{n=1}^{\infty},\left\{B_{n}\right\}_{n=1}^{\infty} \in \mathfrak{M}$, the convex combination, $\alpha\left\{A_{n}\right\}_{n=1}^{\infty}+(1-\alpha)\left\{B_{n}\right\}_{n=1}^{\infty}$, is also an element of $\mathfrak{M}$ and if one of them is $B_{0}$-normal with respect to $f$, then the sequence $\alpha\left\{A_{n}\right\}_{n=1}^{\infty}+$ $(1-\alpha)\left\{B_{n}\right\}_{n=1}^{\infty}$ is also $B_{0}$-normal with respect to $f$. Each $B_{0}$-normal sequence of mappings with respect to $f$ is, in particular, $B_{0}$-weakly normal with respect to $f$, but not vice versa as is shown in the following example.

Example 1.2. Let $X=\mathbb{R}$ and $K=[0, \infty)$. Let $B_{0} \in \mathfrak{B}$ be defined by $B_{0}(x):=\sin ^{2} x$ for each $x \in K$. For each positive integer $n$, define $A_{n}: K \rightarrow K$ by

$$
A_{n} x:= \begin{cases}\left(1-n^{-2^{-1}}\right)^{2^{-1}} x, & x \leq 1, \\ \left(3-2\left(1-n^{-2^{-1}}\right)^{2^{-1}}\right) x+3\left(\left(1-n^{-2^{-1}}\right)^{2^{-1}}-1\right), & 1<x \leq 3 \cdot 2^{-1} \\ x, & 3 \cdot 2^{-1}<x \leq 2 \\ 2, & x>2,\end{cases}
$$

for each $x \in K$. Let $f: K \rightarrow \mathbb{R}$ be defined by $f(x):=x^{2}$ for each $x \in K$. It is clear that $f$ is convex and uniformly continuous on bounded sets. Let $\varepsilon>0$ be arbitrary. For each positive integer $n$, set $\delta_{n}:=n^{-2^{-1}} \varepsilon$. Then $\inf (f)=0$, and for each $x \in S_{B_{0}}$ such that $f(x) \geq \varepsilon$ and each $k=1,2, \ldots, n$, we have

$$
f\left(A_{k} x\right)=\left(1-k^{-2^{-1}}\right) f(x) \leq f(x)-k^{-2^{-1}} \varepsilon=f(x)-\delta_{k} \leq f(x)-\delta_{n} .
$$

Clearly, $\lim _{n \rightarrow \infty} n \delta_{n}=\infty$. Therefore the sequence $\left\{A_{n}\right\}_{n=1}^{\infty}$ is $B_{0}$-weakly normal with respect to $f$, but it is not $B_{0}$-normal with respect to $f$ because $\lim _{n \rightarrow \infty} f\left(A_{n} x\right)=f(x)$ for each $x \in S_{B_{0}}$ such that $f(x) \geq \varepsilon$. As a matter of fact, we also have $\left\{A_{n}\right\}_{n=1}^{\infty} \in \mathfrak{M}_{c}$, that is, $\mathfrak{M}_{c} \subset \mathfrak{M} \neq \emptyset$.

In the sequel, we assume that the function $f$ is clearly understood, and therefore we use the notions of the operator-dependent normality and the operator-dependent weak normality without referring explicitly to $f$.

The importance of concepts related to normality and weak normality for convex minimization problems was plainly demonstrated in [2], [3] and [4], where the function $f$ is uniformly continuous on the set $K$. In this paper, we present analogous results under different assumptions. In contrast with previous studies, here the concepts of normality and weak normality are considered with respect to a given operator $B_{0} \in \mathfrak{B}$ and not globally, and the residual sets depend on the operator $B_{0}$. This operator-dependent approach turns out to be useful for removing the somewhat restrictive requirement on the function $f$ to be uniformly continuous on all of 
$K$. We also present numerous applications of our results to generic minimization problems on arbitrary closed balls.

The rest of our paper is organized as follows. In Section 2, we state our main results. Several auxiliary results are presented in Section 3. Section 4 is devoted to the results concerning the existence of residual sets of operator-dependent normal mappings, operator-dependent normal sequences and of operator-dependent weakly normal sequences of mappings. In Section 5, we provide some applications of operator-dependent normality and operator-dependent weak normality to the study of certain minimization problems. The proofs of our main results, which are stated in Section 2, are provided in Section 6. Finally, in Section 7, we present a few corollaries of these results, which involve metric projections onto closed balls.

In all our results, we also assume that $(X,\|\cdot\|)$ is a Banach space.

\section{STATEMENTS OF THE MAIN RESULTS}

In this section we state our three main results. We establish them in Section 6 below.

Theorem 2.1. Let $B_{0} \in \mathfrak{B}$. Then there exist sets $\mathscr{F} \subset \mathfrak{M}, \mathscr{F}_{b} \subset \mathscr{F} \cap \mathfrak{M}_{b}, \mathscr{F}_{c} \subset \mathscr{F} \cap \mathfrak{M}_{c}$ and $\mathscr{F}_{b c} \subset \mathscr{F}_{b} \cap \mathfrak{M}_{c}$ of $B_{0}$-weakly normal sequences of mappings, which are countable intersections of open (in the relative weak topology) and dense (respectively, in the weak topology, in the relative strong topology, in the relative weak topology and in the relative strong topology) sets in, respectively, $\mathfrak{M}, \mathfrak{M}_{b}, \mathfrak{M}_{c}$ and $\mathfrak{M}_{b c}$ such that for each $\left\{A_{n}\right\}_{n=1}^{\infty} \in \mathscr{F}$, the following assertion holds:

For each $\varepsilon>0$, there exist a neighborhood $U$ (in the weak topology) of $\left\{A_{n}\right\}_{n=1}^{\infty}$ and a positive integer $N$ satisfying

$$
f\left(B_{N} \ldots B_{1} B_{0} x\right)<\inf (f)+\varepsilon
$$

for each $\left\{B_{n}\right\}_{n=1}^{\infty} \in U$ and each $x \in K$.

Theorem 2.2. Let $B_{0} \in \mathfrak{B}$. Then there exist a set $\mathscr{F} \subset \mathfrak{A}$ of $B_{0}$-normal mappings, which is a countable intersection of open and dense sets in $\mathfrak{A}$, and a set $\mathscr{F}_{c} \subset \mathscr{F} \cap \mathfrak{A}_{c}$ of $B_{0}$-normal mappings, which is a countable intersection of open and dense sets in $\mathfrak{A}_{c}$, such that for each $A \in \mathscr{F}$, the following assertion holds:

For each $\varepsilon>0$, there exist a neighborhood $U$ of $A$ in $\mathfrak{A}$ and a positive integer $N$ satisfying

$$
f\left(B^{N} B_{0} x\right)<\inf (f)+\varepsilon
$$

for each $B \in U$ and each $x \in K$.

Theorem 2.3. Let $B_{0} \in \mathfrak{B}$. Then there exist sets $\mathscr{F}_{b} \subset \mathfrak{M}_{b}$ and $\mathscr{F}_{b c} \subset \mathscr{F}_{b} \cap \mathfrak{M}_{c}$ of $B_{0}$-normal sequences of mappings, which are countable intersections of open (in the relative strong topology) and dense (in the relative strong topology) sets in, respectively, $\mathfrak{M}_{b}$ and $\mathfrak{M}_{b c}$, such that for each $\left\{A_{n}\right\}_{n=1}^{\infty} \in \mathscr{F}$, the following assertion holds:

For each $\varepsilon>0$, there exist a neighborhood $U$ (in the strong topology) of $\left\{A_{n}\right\}_{n=1}^{\infty}$ and a positive integer $N$ satisfying

$$
f\left(B_{r(N)} \ldots B_{r(1)} B_{0} x\right)<\inf (f)+\varepsilon
$$

for each $\left\{B_{n}\right\}_{n=1}^{\infty} \in U$, each mapping $r:\{1,2, \ldots\} \rightarrow\{1,2, \ldots\}$ and each $x \in K$.

These theorems generalize the corresponding results in [3] and [4]. 


\section{AUXiLiary RESUlts}

We first prove that there exists an operator $A_{*}: K \rightarrow K$, which is continuous and $B_{0}$-normal for each $B_{0} \in \mathfrak{B}$.

Proposition 3.1. There exists an operator $A_{*} \in \mathfrak{A}_{c}$, which is $B_{0}$-normal for each $B_{0} \in \mathfrak{B}$.

Proof. Given $\varepsilon>0$, there exists a positive integer $j$ such that $2^{-j}<\varepsilon$. Without loss of generality, we may assume that $f$ does not attain its minimum on $K$. Therefore, there exists a function $p: K \rightarrow \mathbb{N}$ such that, for each $x \in K$,

$$
p(x)=\min \left\{i \in \mathbb{N}: f(x)>\inf (f)+2^{-i}\right\} .
$$

Clearly, there exists an open cover $\left\{V_{x}\right\}_{x \in K}$ of $K$ such that, for each $x \in K$,

$$
\left(\forall y \in V_{x}\right) f(y)>\inf (f)+2^{-p(x)} .
$$

For each $x \in K$, by (3.1) and (3.2), we have

$$
\left(\forall y \in V_{x}\right) \quad p(y) \leq p(x)
$$

and there exists $a_{x} \in K$ such that

$$
f\left(a_{x}\right)<\inf (f)+2^{-p(x)-1} .
$$

Since metric spaces are paracompact Hausdorff spaces [5], there is a continuous partition of unity $\left\{\phi_{x}\right\}_{x \in K}$ subordinated to the cover $\left\{V_{x}\right\}_{x \in K}$. That is, for every $x \in K, \phi_{x}: K \rightarrow[0,1]$ is a continuous function such that $\operatorname{supp}\left(\phi_{x}\right) \subset V_{x}$ and for each $x \in K$, there is a neighborhood $U_{x}$ of $x$ and a finite sequence $\left\{x_{i}^{x}\right\}_{i=1}^{m_{x}} \subset K$ such that

$$
\left(\forall y \in U_{x}\right) \sum_{i=1}^{m_{x}} \phi_{x_{i}^{x}}(y)=1 \text { and }\left\{z \in X: \phi_{z}(y)>0\right\} \subset\left\{x_{i}^{x}\right\}_{i=1}^{m_{x}} .
$$

Since $K$ is convex, the definition of a partition of unity implies that there exists a mapping $A_{*}: K \rightarrow K$ defined by $(\forall x \in K) A_{*} x:=\sum_{z \in K} \phi_{z}(z) a_{z}$. Since for each $x \in K$ and each $y \in U_{x}$, we have by (3.5),

$$
A_{*} y=\sum_{i=1}^{m_{x}} \phi_{x_{i}^{x}}(y) a_{x_{i}^{x}}
$$

Since $\phi_{x_{i}^{x}}$ is continuous for each $i=1, \ldots m_{x}$, it follows that $A_{*}$ is also continuous on $K$. Let $x \in K$ satisfy $f(x) \geq \inf (f)+\varepsilon$. Then by (3.1), $p(x) \leq j$. Since $f$ is a convex function, it follows from (3.5) and (3.6) that

$$
f\left(A_{*} x\right) \leq \max \left\{f\left(a_{x_{i}^{x}}\right): i \in\left\{1 \ldots m_{x}\right\}\right\} .
$$

Evidently, we may assume that $\phi_{x_{i}^{x}}(x) \neq 0$ for all $i \in\left\{1 \ldots m_{x}\right\}$. Therefore, for each $i \in$ $\left\{1 \ldots m_{x}\right\}$, we have $x \in \operatorname{supp}\left(\phi_{x_{i}^{x}}\right) \subset V_{x_{i}^{x}}$. Now, by (3.3) and (3.4), we have

$$
f\left(a_{x_{i}^{x}}\right)<\inf (f)+2^{-p\left(x_{i}^{x}\right)-1} \leq \inf (f)+2^{-p(x)-1}
$$

for each $i \in\left\{1 \ldots m_{x}\right\}$. Using (3.7), we obtain that $f\left(A_{*} x\right)<\inf (f)+2^{-p(x)-1}$. On the other hand, by (3.1), $\inf (f)<f(x)-2^{-p(x)}$ and therefore

$$
f\left(A_{*} x\right)<f(x)-2^{-p(x)-1} .
$$


Now, we choose $\delta(\varepsilon)=2^{-j-1}$. Since $p(x) \leq j$, it follows from (3.8) that $A_{*}$ is $B_{0}$-normal for each $B_{0} \in \mathfrak{B}$ and satisfies (1.2). In the case where $K$ is unbounded, since $\lim _{x \rightarrow \infty} f(x)=\infty$, it follows from (3.4) that $\left\{a_{x}: x \in K\right\}$ is bounded. Using (3.6), we conclude that $A_{*} \in \mathfrak{A}_{c}$ even if $K$ is unbounded, as asserted.

Note that, in contrast with [2], in the above proof we use the concept of a partition of unity and not Michael's selection theorem [6].

Lemma 3.1. Let $B_{0} \in \mathfrak{A}$, let $\left\{A_{n}\right\}_{n=1}^{\infty} \in \mathfrak{M}$ be a $B_{0}$-normal sequence and let $\varepsilon>0$ be given. Then there exist a number $\delta>0$ and a neighborhood $U$ of $\left\{A_{n}\right\}_{n=1}^{\infty}$ in $\mathfrak{M}$ with the strong topology such that, for each $\left\{B_{n}\right\}_{n=1}^{\infty} \in U$ and each $x \in S_{B_{0}}$ satisfying $f(x) \geq \inf (f)+\varepsilon$, $f\left(B_{n} x\right)<f(x)-\delta$ for each $n=1,2, \ldots$

Proof. Since $\left\{A_{n}\right\}_{n=1}^{\infty}$ is $B_{0}$-normal, there is $\delta_{0}>0$ such that, for each $n=1,2, \ldots$ and each $x \in S_{B_{0}}$ satisfying $f(x) \geq \inf (f)+\varepsilon$, we have

$$
f\left(A_{n} x\right)<f(x)-\delta_{0} .
$$

Since $f$ is uniformly continuous on $S_{B_{0}}$, there is $\delta \in\left(0,2^{-1} \delta_{0}\right)$ such that $|f(y)-f(z)|<2^{-1} \delta_{0}$ for each $y, z \in S_{B_{0}}$ satisfying $\|y-z\|<\delta$. Set

$$
U:=\left\{\left\{B_{n}\right\}_{n=1}^{\infty} \in \mathfrak{M}:\left(\left\{A_{n}\right\}_{n=1}^{\infty},\left\{B_{n}\right\}_{n=1}^{\infty}\right) \in E_{2}(\delta)\right\} .
$$

It is clear that $U$ is a neighborhood of $\left\{A_{n}\right\}_{n=1}^{\infty}$ in $\mathfrak{M}$ with the strong topology. Assume that $\left\{B_{n}\right\}_{n=1}^{\infty} \in U$ and that $x \in S_{B_{0}}$ satisfies $f(x) \geq \inf (f)+\varepsilon$. Then by (3.9), we have

$$
f\left(A_{n} x\right)<f(x)-\delta_{0}
$$

for each $n=1,2, \ldots$. The definitions of $\delta$ and $U$ imply that

$$
\left\|A_{n} x-B_{n} x\right\|<\delta
$$

and

$$
\left|f\left(A_{n} x\right)-f\left(B_{n} x\right)\right|<2^{-1} \delta_{0}
$$

for each $n=1,2, \ldots$. When combined with (3.10), this implies that

$$
f\left(B_{n} x\right)<f(x)+2^{-1} \delta_{0}-\delta_{0}<f(x)-\delta
$$

for each $n=1,2, \ldots$, as asserted.

Lemma 3.2. Let $B_{0} \in \mathfrak{B}$, let $\left\{A_{n}\right\}_{n=1}^{\infty} \in \mathfrak{M}$ be a $B_{0}$-weakly normal sequence and let $\varepsilon>0$ be given. Then there exist a sequence of positive numbers $\left\{\delta_{N}\right\}_{N=1}^{\infty}$ and a sequence $\left\{U_{N}\right\}_{N=1}^{\infty}$ of neighborhoods of $\left\{A_{n}\right\}_{n=1}^{\infty}$ in $\mathfrak{M}$ with the weak topology such that $\lim _{\sup } \operatorname{su}_{N \rightarrow \infty} N \delta_{N}=\infty$ and for each positive integer $N$, the following assertion holds:

For each $\left\{B_{n}\right\}_{n=1}^{\infty} \in U_{N}$ and each $x \in S_{B_{0}}$ satisfying $f(x) \geq \inf (f)+\varepsilon$, we have $f\left(B_{n} x\right)<$ $f(x)-\delta_{N}$ for each $n=1,2, \ldots, N$.

Proof. Since $\left\{A_{n}\right\}_{n=1}^{\infty}$ is $B_{0}$-weakly normal, there is a sequence $\left\{\delta_{N}^{\prime}\right\}_{N=1}^{\infty}$ of positive numbers such that $\lim \sup _{N \rightarrow \infty} N \delta_{N}^{\prime}=\infty$ and for each $x \in S_{B_{0}}$ satisfying $f(x) \geq \inf (f)+\varepsilon$, we have

$$
f\left(A_{n} x\right)<f(x)-\delta_{N}^{\prime}
$$

for all $N=1,2 \ldots$ and each $n=1,2, \ldots, N$. 
Let $N$ be a positive integer. Set $\delta_{N}:=2^{-1} \delta_{N}^{\prime}$. Since $f$ is uniformly continuous on $S_{B_{0}}$, there is a number $\delta_{N}^{\prime \prime}>0$ such that $|f(y)-f(z)|<\delta_{N}$ for each $y, z \in S_{B_{0}}$ satisfying $\|y-z\|<\delta_{N}^{\prime \prime}$. Set

$$
U_{N}:=\left\{\left\{B_{n}\right\}_{n=1}^{\infty} \in \mathfrak{M}:\left(\left\{A_{n}\right\}_{n=1}^{\infty},\left\{B_{n}\right\}_{n=1}^{\infty}\right) \in E_{1}\left(N, \delta_{N}^{\prime \prime}\right)\right\} .
$$

Clearly, $U_{N}$ is a neighborhood of $\left\{A_{n}\right\}_{n=1}^{\infty}$ in $\mathfrak{M}$ with the weak topology. Assume that $\left\{B_{n}\right\}_{n=1}^{\infty} \in$ $U_{N}$ and that $x \in S_{B_{0}}$ satisfies $f(x) \geq \inf (f)+\varepsilon$. Then, it follows from (3.11) that

$$
f\left(A_{n} x\right)<f(x)-\delta_{N}^{\prime}
$$

for each $n=1,2, \ldots, N$. The definitions of $\delta_{N}^{\prime \prime}$ and $U_{N}$ imply that $\left\|A_{n} x-B_{n} x\right\|<\delta_{N}^{\prime \prime}$ and $\left|f\left(A_{n} x\right)-f\left(B_{n} x\right)\right|<\delta_{N}$ for each $n=1,2, \ldots, N$. When combined with (3.12), this implies that

$$
f\left(B_{n} x\right)<f(x)+\delta_{N}-\delta_{N}^{\prime}=f(x)-\delta_{N}
$$

for each $n=1,2, \ldots, N$. In this way, we have constructed two sequences $\left\{\delta_{N}\right\}_{N=1}^{\infty}$ and $\left\{U_{N}\right\}_{N=1}^{\infty}$. Using the $B_{0}$-weak normality of $\left\{A_{n}\right\}_{n=1}^{\infty}$ and the definition of $\left\{\delta_{N}\right\}_{N=1}^{\infty}$, we have

$$
\limsup _{N \rightarrow \infty} N \delta_{N}=\infty
$$

Hence, we see that the sequences $\left\{\delta_{N}\right\}_{N=1}^{\infty}$ and $\left\{U_{N}\right\}_{N=1}^{\infty}$ enjoy all the asserted properties.

Let $B_{0} \in \mathfrak{B}$, let $A_{*}$ be the mapping the existence of which is guaranteed by Proposition 3.1, and let $\left\{A_{n}\right\}_{n=1}^{\infty}$ be an arbitrary sequence in $\mathfrak{M}$. For each $\gamma \in(0,1)$, we define a sequence of mappings $\left\{A_{n}^{\gamma}\right\}_{n=1}^{\infty}, A_{n}^{\gamma}: K \rightarrow K$, by

$$
A_{n}^{\gamma}:=(1-\gamma) A_{n}+\gamma A_{*}, n=1,2, \ldots
$$

By Proposition 3.1 and Remark 1.1, the sequence $\left\{A_{n}^{\gamma}\right\}_{n=1}^{\infty} \in \mathfrak{M}$. For each $\gamma \in(0,1)$ and for each $N=1,2 \ldots$, we have

$$
(\forall n \in\{1, \ldots, N\}) \rho\left(A_{n}^{\gamma}, A_{n}\right) \leq 2 \gamma \max \left\{\max \left\{\sup _{x \in K}\left\|A_{k} x\right\|\right\}_{k=1}^{N}, \sup _{x \in K}\left\|A_{*} x\right\|\right\} .
$$

If, in addition, $\left\{A_{n}\right\}_{n=1}^{\infty} \in \mathfrak{M}_{b}$, then

$$
(\forall n \in\{1,2 \ldots\}) \rho\left(A_{n}^{\gamma}, A_{n}\right) \leq 2 \gamma \max \left\{\sup \left\{\sup _{x \in K}\left\|A_{k} x\right\|\right\}_{k=1}^{\infty}, \sup _{x \in K}\left\|A_{*} x\right\|\right\},
$$

where $\sup \left\{\sup _{x \in K}\left\|A_{k} x\right\|\right\}_{k=1}^{\infty}<\infty$. For an arbitrary operator $A \in \mathfrak{A}$, we define

$$
A^{\gamma}:=(1-\gamma) A+\gamma A_{*} \text {. }
$$

Evidently,

$$
\rho\left(A^{\gamma}, A\right) \leq 2 \gamma \max \left\{\sup _{x \in K}\|A x\|, \sup _{x \in K}\left\|A_{*} x\right\|\right\} .
$$

Lemma 3.3. Let $B_{0} \in \mathfrak{B}$. Then, for each $\varepsilon>0$, there exist a positive number $\delta$ such that, for each $\left\{A_{n}\right\}_{n=1}^{\infty} \in \mathfrak{M}$ and each $\gamma \in(0,1)$, there is a sequence $\left\{U_{N}\right\}_{N=1}^{\infty}$ of neighborhoods of $\left\{A_{n}^{\gamma}\right\}_{n=1}^{\infty}$ in $\mathfrak{M}$ with the weak topology satisfying, for each positive integer $N$, the following assertion: For each $\left\{B_{n}\right\}_{n=1}^{\infty} \in U_{N}$ and each $x \in S_{B_{0}}$ such that $f(x) \geq \inf (f)+\varepsilon$, we have $f\left(B_{n} x\right)<f(x)-\gamma \delta$ for each $n=1,2, \ldots, N$. 
Proof. Let $\varepsilon>0$. Since $A_{*}$ is $B_{0}$-normal, there is a positive number $\delta^{\prime}$ such that, for each $x \in S_{B_{0}}$ satisfying $f(x) \geq \inf (f)+\varepsilon$, we have

$$
f\left(A_{*} x\right)<f(x)-\delta^{\prime} .
$$

Set $\delta:=2^{-1} \delta^{\prime}$. Let $\left\{A_{n}\right\}_{n=1}^{\infty} \in \mathfrak{M}$ and $\gamma \in(0,1)$. The convexity of $f$ implies that

$$
f\left(A_{n}^{\gamma} x\right)<f(x)-\gamma \delta^{\prime}
$$

for each $n=1,2 \ldots$ Let $N$ be a positive integer. Since $f$ is uniformly continuous on $S_{B_{0}}$, there is a number $\delta^{\prime \prime}>0$ such that $|f(y)-f(z)|<\gamma \delta$ for each $y, z \in S_{B_{0}}$ satisfying $\|y-z\|<\delta^{\prime \prime}$. Set

$$
U_{N}:=\left\{\left\{B_{n}\right\}_{n=1}^{\infty} \in \mathfrak{M}:\left(\left\{A_{n}^{\gamma}\right\}_{n=1}^{\infty},\left\{B_{n}\right\}_{n=1}^{\infty}\right) \in E_{1}\left(N, \delta^{\prime \prime}\right)\right\} .
$$

Clearly, $U_{N}$ is a neighborhood of $\left\{A_{n}^{\gamma}\right\}_{n=1}^{\infty}$ in $\mathfrak{M}$ with the weak topology. Assume that $\left\{B_{n}\right\}_{n=1}^{\infty} \in$ $U_{N}$ and that $x \in S_{B_{0}}$ satisfies $f(x) \geq \inf (f)+\varepsilon$. The definitions of $\delta^{\prime \prime}$ and $U_{N}$ imply that $\left\|A_{n} x-B_{n} x\right\|<\delta^{\prime \prime}$ and $\left|f\left(A_{n}^{\gamma} x\right)-f\left(B_{n} x\right)\right|<\gamma \delta$ for each $n=1,2, \ldots, N$. When combined with (3.18), this implies that

$$
f\left(B_{n} x\right)<f(x)+\gamma \delta-\gamma \delta^{\prime}=f(x)-\gamma \delta
$$

for each $n=1,2, \ldots, N$. In this way, we have found a number $\delta$ and constructed a sequence of neighborhoods $\left\{U_{N}\right\}_{N=1}^{\infty}$ which have all the asserted properties.

\section{RESIDUAL SETS OF OPERATOR-DEPENDENT NORMAL MAPPINGS, OPERATOR-DEPENDENT NORMAL SEQUENCES OF MAPPINGS AND OF OPERATOR-DEPENDENT WEAKLY NORMAL SEQUENCES OF MAPPINGS}

Recall that a subset $Z$ of a topological space $Y$ is called residual if it contains a countable intersection of open and dense subsets of $Y$. In the case where the space $Y$ is completely pseudo-metrizable, the Baire category theorem guarantees that $Z$ is also a dense subset of $Y$. In this section, we prove that there exist residual sets of operator-dependent normal mappings, operator-dependent normal sequences of mappings and operator-dependent weakly normal sequences of mappings. More examples of applications of the concept of genericity to nonlinear analysis can be found, for example, in [7].

Theorem 4.1. For each $B_{0} \in \mathfrak{B}$, there exist sets $\mathscr{F} \subset \mathfrak{M}, \mathscr{F}_{b} \subset \mathscr{F} \cap \mathfrak{M}_{b}, \mathscr{F}_{c} \subset \mathscr{F} \cap \mathfrak{M}_{c}$ and $\mathscr{F}_{b c} \subset \mathscr{F}_{b} \cap \mathfrak{M}_{c}$, which are countable intersections of open (in the relative weak topology) and dense (respectively, in the weak topology, in the relative strong topology, in the relative weak topology and in the relative strong topology) sets in, respectively, $\mathfrak{M}, \mathfrak{M}_{b}, \mathfrak{M}_{c}$ and $\mathfrak{M}_{b c}$ such that each sequence $\left\{B_{n}\right\}_{n=1}^{\infty} \in \mathscr{F}$ is $B_{0}$-weakly normal.

Proof. Define

$$
T:=\left\{\gamma \in(0,1): \gamma=N^{-2^{-1}} \text { for some positive integer } N\right\},
$$

and for each positive integer $N$ define

$$
T_{N}:=\left\{\gamma \in T: \gamma<N^{-2^{-1}}\right\} .
$$

By (3.14) and Proposition 3.1, for each $N=1,2 \ldots$, the set

$$
A^{N}=\left\{\left\{A_{n}^{\gamma}\right\}_{n=1}^{\infty}:\left\{A_{n}\right\}_{n=1}^{\infty} \in \mathfrak{M}, \gamma \in T_{N}\right\}
$$


is dense in $\mathfrak{M}$ with the weak topology, and the set

$$
A_{c}^{N}=\left\{\left\{A_{n}^{\gamma}\right\}_{n=1}^{\infty}:\left\{A_{n}\right\}_{n=1}^{\infty} \in \mathfrak{M}_{c}, \gamma \in T_{N}\right\}
$$

is dense in $\mathfrak{M}_{c}$ with the relative weak topology. By (3.15) and Proposition 3.1, for each $N=$ $1,2 \ldots$, the set

$$
A_{b}^{N}=\left\{\left\{A_{n}^{\gamma}\right\}_{n=1}^{\infty}:\left\{A_{n}\right\}_{n=1}^{\infty} \in \mathfrak{M}_{b}, \gamma \in T_{N}\right\}
$$

is dense in $\mathfrak{M}_{b}$ with the relative strong topology, and the set

$$
A_{b c}^{N}=\left\{\left\{A_{n}^{\gamma}\right\}_{n=1}^{\infty}:\left\{A_{n}\right\}_{n=1}^{\infty} \in \mathfrak{M}_{b c}, \gamma \in T_{N}\right\}
$$

is dense in $\mathfrak{M}_{b c}$ with the relative strong topology.

Let $q$ be an arbitrary positive integer. By Lemma 3.3, there exist a number $\delta(q)>0$ such that, for each $\left(\left\{A_{n}\right\}_{n=1}^{\infty}, \gamma\right) \in \mathfrak{M} \times T$, there is a sequence $\left\{U_{N}\left(\left\{A_{n}\right\}_{n=1}^{\infty}, \gamma\right)(q)\right\}_{N=1}^{\infty}$ of open neighborhoods of $\left\{A_{n}^{\gamma}\right\}_{n=1}^{\infty}$ with the weak topology satisfying, for each positive integer $N$, the following assertion:

For each $\left\{B_{n}\right\}_{n=1}^{\infty} \in U_{N}\left(\left\{A_{n}\right\}_{n=1}^{\infty}, \gamma\right)(q)$ and each $x \in S_{B_{0}}$ satisfying $f(x) \geq \inf (f)+2^{-q}$, we have

$$
f\left(B_{n} x\right)<f(x)-\gamma \delta(q)
$$

for each $n=1,2 \ldots, N$.

For each pair of positive integers $q$ and $N$, set

$$
\begin{aligned}
& \mathscr{D}_{q, N}:=\cup_{\left(\left\{A_{n}\right\}_{n=1}^{\infty}, \gamma\right) \in \mathfrak{M} \times T_{N}}\left(U_{\gamma^{-2}}\left(\left\{A_{n}\right\}_{n=1}^{\infty}, \gamma\right)(q)\right), \\
& \mathscr{D}_{q, N}^{b}::=\cup_{\left(\left\{A_{n}\right\}_{n=1}^{\infty}, \gamma\right) \in \mathfrak{M}_{b} \times T_{N}} U_{\gamma^{-2}}\left(\left\{A_{n}\right\}_{n=1}^{\infty}, \gamma\right)(q) \cap \mathfrak{M}_{b}, \\
& \mathscr{D}_{q, N}^{c}::=\cup_{\left(\left\{A_{n}\right\}_{n=1}^{\infty}, \gamma\right) \in \mathfrak{M}_{c} \times T_{N}} U_{\gamma^{-2}}\left(\left\{A_{n}\right\}_{n=1}^{\infty}, \gamma\right)(q) \cap \mathfrak{M}_{c}, \\
& \text { and } \\
& \mathscr{D}_{q, N}^{b c}:=\cup_{\left(\left\{A_{n}\right\}_{n=1}^{\infty}, \gamma\right) \in \mathfrak{M}_{b c} \times T_{N}} U_{\gamma^{-2}}\left(\left\{A_{n}\right\}_{n=1}^{\infty}, \gamma\right)(q) \cap \mathfrak{M}_{b c} .
\end{aligned}
$$

Clearly, the sets $\mathscr{D}_{q, N}, \mathscr{D}_{q, N}^{b}, \mathscr{D}_{q, N}^{c}$ and $\mathscr{D}_{q, N}^{b c}$ are open (in the relative weak topology) and dense (respectively, in the weak topology, in the relative strong topology, in the relative weak topology and in the relative strong topology) sets in, respectively, $\mathfrak{M}, \mathfrak{M}_{b}, \mathfrak{M}_{c}$ and $\mathfrak{M}_{b c}$ for each pair of positive integers $q$ and $N$, because these sets contain, respectively, $A^{N}, A_{b}^{N}, A_{c}^{N}$ and $A_{b c}^{N}$. Define $\mathscr{F}=\cap_{q=1}^{\infty} \cap_{N=1}^{\infty} \mathscr{D}_{q, N}, \mathscr{F}_{b}=\cap_{q=1}^{\infty} \cap_{N=1}^{\infty} \mathscr{D}_{q, N}^{b}, \mathscr{F}_{c}=\cap_{q=1}^{\infty} \cap_{N=1}^{\infty} \mathscr{D}_{q, N}^{c}$ and $\mathscr{F}_{b c}=\cap_{q=1}^{\infty} \cap_{N=1}^{\infty}$ $\mathscr{D}_{q, N}^{b c}$. Evidently, $\mathscr{F}, \mathscr{F}_{b}, \mathscr{F}_{c}$ and $\mathscr{F}_{b c}$ are countable intersections of open (in the relative weak topology) and dense (respectively, in the weak topology, in the relative strong topology, in the relative weak topology and in the relative strong topology) sets in, respectively, $\mathfrak{M}, \mathfrak{M}_{b}, \mathfrak{M}_{c}$ and $\mathfrak{M}_{b c}$.

Assume now that $\left\{B_{n}\right\}_{n=1}^{\infty} \in \mathscr{F}$ and let $\varepsilon>0$ be an arbitrary positive number. Choose a positive integer $q_{0}$ such that $2^{-q_{0}}<\varepsilon$. Then, for each positive integer $N$, there exists a pair $\left(\left\{A_{n}\right\}_{n=1}^{\infty}, \gamma_{N}\right) \in \mathfrak{M} \times T_{N}$ such that

$$
\left\{B_{n}\right\}_{n=1}^{\infty} \in U_{\gamma_{N}^{-2}}\left(\left\{A_{n}\right\}_{n=1}^{\infty}, \gamma_{N}\right)\left(q_{0}\right) .
$$

It follows from (4.1) that, for each point $x \in S_{B_{0}}$ satisfying $f(x) \geq \inf (f)+\varepsilon$,

$$
f\left(B_{n} x\right)<f(x)-\gamma_{N} \delta\left(q_{0}\right)
$$

for each $n=1,2 \ldots, \gamma_{N}^{-2}$. 
Consider the sequences $\left\{\gamma_{N}\right\}_{N=1}^{\infty}$ and $\left\{\gamma_{N}^{-2}\right\}_{N=1}^{\infty}$. Since for each positive integer $N$ we have $\gamma_{N}^{-2}>N$, it is clear that there exists a strictly increasing subsequence $\left\{\gamma_{N_{k}}^{-2}\right\}_{k=1}^{\infty}$ of $\left\{\gamma_{N}^{-2}\right\}_{N=1}^{\infty}$. For each positive integer $M$, set $\delta_{M}:=\gamma_{N}{ }_{\min \left\{k: \gamma_{N_{k}}^{2} \geq M\right.}$.

Since $\gamma_{N \text { min }\left\{k: \gamma_{N_{k}}^{-2} \geq M\right\}}^{-2} \geq M$, we conclude from (4.2) that, for each point $x \in S_{B_{0}}$ satisfying $f(x) \geq \inf (f)+\varepsilon$,

$$
f\left(B_{n} x\right)<f(x)-\delta_{M} \delta\left(q_{0}\right)
$$

for each $n=1,2 \ldots, M$. Since for each $k=1,2 \ldots$, we have

$$
\delta_{\gamma_{N_{k}}^{-2}}=\gamma_{\min \left\{i: \gamma_{N_{i}}^{-2} \geq \gamma_{N_{k}}^{-2}\right\}}=\gamma_{N_{k}}
$$

it follows that

$$
\lim _{n \rightarrow \infty} \gamma_{N_{k}}^{-2} \delta_{\gamma_{N_{k}}^{-2}}=\lim _{n \rightarrow \infty} \gamma_{N_{k}}^{-1}=\infty
$$

Hence $\left\{B_{n}\right\}_{n=1}^{\infty}$ is $B_{0}$-weakly normal. This completes the proof of Theorem 4.1.

Theorem 4.2. For each $B_{0} \in \mathfrak{B}$, there exist a set $\mathscr{F} \subset \mathfrak{M}_{b}$, which is a countable intersection of open and dense sets in $\mathfrak{A}$, and a set $\mathscr{F}_{c} \subset \mathscr{F} \cap \mathfrak{A}_{c}$, which is a countable intersection of open and dense sets in $\mathfrak{A}_{c}$ such that each mapping $B \in \mathscr{F}$ is $B_{0}$-normal.

Proof. By (3.17) and Proposition 3.1, the set

$$
A=\left\{A^{\gamma}: A \in \mathfrak{A}, \gamma \in(0,1)\right\}
$$

is dense in $\mathfrak{A}$, and the set

$$
A_{c}=\left\{A^{\gamma}: A \in \mathfrak{A}_{c}, \gamma \in(0,1)\right\}
$$

is dense in $\mathfrak{A}_{c}$.

By Remark 1.1, for each $A \in \mathfrak{A}$ and each $\gamma \in(0,1)$, the mapping $A^{\gamma}$ is $B_{0}$-normal. Assume that $q$ is an arbitrary positive integer. By Lemma 3.1, for each $(A, \gamma) \in \mathfrak{A} \times(0,1)$, there exist a number $\delta_{q}(A, \gamma)>0$ and an open neighborhood $U_{q}(A, \gamma)$ of $A^{\gamma}$ in $\mathfrak{A}$ such that the following assertion holds:

For each $B \in U_{q}(A, \gamma)$ and each $x \in S_{B_{0}}$ satisfying $f(x) \geq \inf (f)+2^{-q}$, we have

$$
f(B x)<f(x)-\delta_{q}
$$

For each positive integer $q$, set

$$
\begin{gathered}
\mathscr{D}_{q}:=\cup_{(A, \gamma) \in \mathfrak{A} \times(0,1)} U_{q}(A, \gamma), \\
\mathscr{D}_{q}^{c}:=\cup_{(A, \gamma) \in \mathfrak{A}_{c} \times(0,1)} U_{q}(A, \gamma) \cap \mathfrak{A}_{c} .
\end{gathered}
$$

It is clear that the sets $\mathscr{D}_{q}$ and $\mathscr{D}_{q}^{c}$ are open and dense sets in, respectively, $\mathfrak{A}$ and $\mathfrak{A}_{c}$ for each $q=1,2 \ldots$, because these sets contain, respectively, $A$ and $A_{c}$. Define $\mathscr{F}=\cap_{q=1}^{\infty} \mathscr{D}_{q}$ and $\mathscr{F}_{c}=\cap_{q=1}^{\infty} \mathscr{D}_{q}^{c}$. Evidently, $\mathscr{F}_{\text {and }} \mathscr{F}_{c}$ are countable intersections of open and dense sets in, respectively, $\mathfrak{A}$ and $\mathfrak{A}_{c}$.

Assume now that $B \in \mathscr{F}$. Let $\varepsilon>0$ be an arbitrary positive number and choose a positive integer $q_{0}$ such that $2^{-q_{0}}<\varepsilon$. There exists a pair $(A, \gamma) \in \mathfrak{A} \times(0,1)$ such that $B \in U_{q_{0}}(A, \gamma)$. It follows from (4.3) that for each point $x \in S_{B_{0}}$ satisfying $f(x) \geq \inf (f)+\varepsilon$, we have

$$
f(B x)<\inf (f)-\delta_{q_{0}} .
$$


Hence $B$ is $B_{0}$-normal. This completes the proof of Theorem 4.2.

Theorem 4.3. For each $B_{0} \in \mathfrak{B}$, there exist sets $\mathscr{F}_{b} \subset \mathscr{F}_{\mathfrak{M}_{b}}$ and $\mathscr{F}_{b c} \subset \mathscr{F}_{b} \cap \mathfrak{M}_{b c}$, which are countable intersections of open (in the relative strong topology) and dense (in the relative strong topology) sets in, respectively, $\mathfrak{M}_{b}$ and $\mathfrak{M}_{b c}$, such that each sequence $\left\{B_{n}\right\}_{n=1}^{\infty} \in \mathscr{F}$ is $B_{0}$-normal.

Proof. By (3.15) and Proposition 3.1, the set

$$
A_{b}=\left\{\left\{A_{n}^{\gamma}\right\}_{n=1}^{\infty}:\left\{A_{n}\right\}_{n=1}^{\infty} \in \mathfrak{M}_{b}, \gamma \in(0,1)\right\}
$$

is dense in $\mathfrak{M}_{b}$ with the relative strong topology and the set

$$
A_{b c}=\left\{\left\{A_{n}^{\gamma}\right\}_{n=1}^{\infty}:\left\{A_{n}\right\}_{n=1}^{\infty} \in \mathfrak{M}_{b c}, \gamma \in(0,1)\right\}
$$

is dense in $\mathfrak{M}_{b c}$ with the relative strong topology.

By Remark 1.1, for each $\left\{A_{n}\right\}_{n=1}^{\infty} \in \mathfrak{M}$ and each $\gamma \in(0,1)$, the sequence $\left\{A_{n}^{\gamma}\right\}_{n=1}^{\infty}$ is $B_{0^{-}}$ normal. Let $q$ be an arbitrary positive integer. By Lemma 3.1, for each pair $\left(\left\{A_{n}\right\}_{n=1}^{\infty}, \gamma\right) \in$ $\mathfrak{M} \times(0,1)$, there exist a number $\delta_{q}\left(\left\{A_{n}\right\}_{n=1}^{\infty}, \gamma\right)>0$ and an open neighborhood $U_{q}\left(\left\{A_{n}\right\}_{n=1}^{\infty}, \gamma\right)$ of $\left\{A_{n}^{\gamma}\right\}_{n=1}^{\infty}$ in $\mathfrak{M}$ with the strong topology such that the following assertion holds:

For each $\left\{B_{n}\right\}_{n=1}^{\infty} \in U_{q}\left(\left\{A_{n}\right\}_{n=1}^{\infty}, \gamma\right)$ and each $x \in S_{B_{0}}$ satisfying $f(x) \geq \inf (f)+2^{-q}$, we have

$$
f\left(B_{n} x\right)<f(x)-\delta_{q}
$$

for each $n=1,2, \ldots$. For each positive integer $q$, set

$$
\begin{aligned}
\mathscr{D}_{q}^{b} & :=\cup_{\left(\left\{A_{n}\right\}_{n=1}^{\infty}, \gamma\right) \in \mathfrak{M}_{b} \times(0,1)} U_{q}\left(\left\{A_{n}\right\}_{n=1}^{\infty}, \gamma\right) \cap \mathfrak{M}_{b}, \\
\mathscr{D}_{q}^{b c} & :=\cup_{\left(\left\{A_{n}\right\}_{n=1}^{\infty}, \gamma\right) \in \mathfrak{M}_{b c} \times(0,1)} U_{q}\left(\left\{A_{n}\right\}_{n=1}^{\infty}, \gamma\right) \cap \mathfrak{M}_{b c} .
\end{aligned}
$$

Clearly, the sets $\mathscr{D}_{q}^{b}$ and $\mathscr{D}_{q}^{b c}$ are open and dense sets in, respectively, $\mathfrak{M}_{b}$ and $\mathfrak{M}_{b c}$ for each $q=1,2 \ldots$, because these sets contain, respectively, $A_{b}$ and $A_{b c}$. Define $\mathscr{F}=\cap_{q=1}^{\infty} \mathscr{D}_{q}^{b}$ and $\mathscr{F}_{b c}=\cap_{q=1}^{\infty} \mathscr{D}_{q}^{b c}$. Evidently, $\mathscr{F}$ and $\mathscr{F}_{c}$ are countable intersections of open and dense sets in, respectively, $\mathfrak{M}_{b}$ and $\mathfrak{M}_{b c}$.

Assume now that $\left\{B_{n}\right\}_{n=1}^{\infty} \in \mathscr{F}$ and let $\varepsilon>0$ be an arbitrary positive number. Choose a positive integer $q_{0}$ such that $2^{-q_{0}}<\varepsilon$. There exists a pair $\left(\left\{A_{n}\right\}_{n=1}^{\infty}, \gamma\right) \in \mathfrak{M}_{b} \times(0,1)$ such that $\left\{B_{n}\right\}_{n=1}^{\infty} \in U_{q_{0}}\left(\left\{A_{n}\right\}_{n=1}^{\infty}, \gamma\right)$. It follows from (4.4) that for each point $x \in S_{B_{0}}$ satisfying $f(x) \geq \inf (f)+\varepsilon$, we have

$$
f\left(B_{n} x\right)<\inf (f)-\delta_{q_{0}}
$$

for each $n=1,2, \ldots$ Hence $\left\{B_{n}\right\}_{n=1}^{\infty}$ is $B_{0}$-normal. This completes the proof of Theorem 4.3.

\section{APPLICATIONS OF OPERATOR-DEPENDENT NORMALITY AND OPERATOR-DEPENDENT WEAK NORMALITY TO THE MINIMIZATION OF CONVEX FUNCTIONS}

In this section we present several applications of the concepts of operator-dependent normality and operator-dependent weak normality to solving certain minimization problems. 
Theorem 5.1. Let $B_{0} \in \mathfrak{B}$, let $\left\{A_{n}\right\}_{n=1}^{\infty} \in \mathfrak{M}$ be $B_{0}$-weakly normal and let $\varepsilon>0$ be given. Then there exist a neighborhood $U$ of $\left\{A_{n}\right\}_{n=1}^{\infty}$ in $\mathfrak{M}$ with the weak topology and a positive integer $N$ such that, for each $\left\{B_{n}\right\}_{n=1}^{\infty} \in U$,

$$
f\left(B_{N} \ldots B_{1} B_{0} x\right)<\inf (f)+\varepsilon
$$

for all $x \in K$.

Proof. Since $\left\{A_{n}\right\}_{n=1}^{\infty}$ is $B_{0}$-weakly normal, employing Lemma 3.2, we see that there exist a positive integer $N$, a positive number $\delta_{N}>0$ satisfying $\delta_{N} N>d_{B_{0}}-\inf (f)$, and a neighborhood $U_{N}$ of $\left\{A_{n}\right\}_{n=1}^{\infty}$ in $\mathfrak{M}$ with the weak topology such that the following assertion holds:

For each $\left\{B_{n}\right\}_{n=1}^{\infty} \in U_{N}$ and each $x \in S_{B_{0}}$ satisfying $f(x) \geq \inf (f)+\varepsilon$, we have

$$
f\left(B_{n} x\right)<f(x)-\delta_{N}
$$

for each $n=1,2, \ldots, N$.

Let $\left\{B_{n}\right\}_{n=1}^{\infty} \in U_{N}$. We claim that

$$
f\left(B_{N} \ldots B_{1} B_{0} x\right)<\inf (f)+\varepsilon
$$

for each $x \in K$. Suppose to the contrary that this is not true. Then there exists $x \in K$ such that

$$
f\left(B_{n} \ldots B_{1} B_{0} x\right) \geq \inf (f)+\varepsilon, n=0, \ldots, N .
$$

From (5.1), it follows by induction that, for each $n=1, \ldots, N$,

$$
f\left(B_{n} \ldots B_{1} B_{0} x\right)<f\left(B_{0} x\right)-n \delta_{N}
$$

This implies that

$$
f\left(B_{N} \ldots B_{1} B_{0} x\right)<f\left(B_{0} x\right)-N \delta_{N}<d_{B_{0}}-\left(d_{B_{0}}-\inf (f)\right)=\inf (f),
$$

a contradiction. Therefore, (5.2) is, in fact, valid and Theorem 5.1 is proved.

Theorem 5.2. Let $B_{0} \in \mathfrak{B}$, let $A \in \mathfrak{A}$ be $B_{0}$-normal and let $\varepsilon>0$ be given. Then there exist a neighborhood $U$ of $A$ in $\mathfrak{A}$ and a positive integer $N$ such that, for each $B \in U$,

$$
f\left(B^{N} B_{0} x\right)<\inf (f)+\varepsilon
$$

for all $x \in K$.

Proof. By Lemma 3.1, there exist a neighborhood $U$ of $A$ in $\mathfrak{A}$ and a number $\delta>0$ such that the following property holds:

For each $B \in U$ and each $x \in S_{B_{0}}$ satisfying $f(x) \geq \inf (f)+\varepsilon$, we have

$$
f(B x)<f(x)-\delta .
$$

Choose a positive integer $N$ such that

$$
\delta N>d_{B_{0}}-\inf (f)
$$

Assume that $B \in U$. We claim that

$$
f\left(B^{N} B_{0} x\right)<\inf (f)+\varepsilon
$$

for each $x \in K$. Suppose to the contrary that this is not true. Then there exists $x \in K$ such that

$$
f\left(B^{n} B_{0} x\right) \geq \inf (f)+\varepsilon, n=0, \ldots, N .
$$


From (5.3), it follows by induction that, for each $n=1, \ldots, N$,

$$
f\left(B^{n} B_{0} x\right)<f\left(B_{0} x\right)-n \delta .
$$

This implies that

$$
f\left(B^{N} B_{0} x\right)<f\left(B_{0} x\right)-N \delta<d_{B_{0}}-\left(d_{B_{0}}-\inf (f)\right)=\inf (f),
$$

a contradiction. Therefore, (5.4) is indeed valid, as claimed, and Theorem 5.2 is proved.

Theorem 5.3. Let $B_{0} \in \mathfrak{B}$, let the sequence $\left\{A_{n}\right\}_{n=1}^{\infty} \in \mathfrak{M}$ be $B_{0}$-normal and let $\varepsilon>0$ be given. Then there exist a neighborhood $U$ of $\left\{A_{n}\right\}_{n=1}^{\infty}$ in $\mathfrak{M}$ with the strong topology and a positive integer $N$ such that, for each $\left\{B_{n}\right\}_{n=1}^{\infty} \in U$ and each mapping $r:\{1,2, \ldots\} \rightarrow\{1,2, \ldots\}$,

$$
f\left(B_{r(N)} \ldots B_{r(1)} B_{0} x\right)<\inf (f)+\varepsilon
$$

for all $x \in K$.

Proof. By Lemma 3.1, there exist a neighborhood $U$ of $\left\{A_{n}\right\}_{n=1}^{\infty}$ in $\mathfrak{M}$ with the strong topology and a number $\delta>0$ such that the following property holds:

For each $\left\{B_{n}\right\}_{n=1}^{\infty} \in U$ and each point $x \in S_{B_{0}}$ satisfying $f(x) \geq \inf (f)+\varepsilon$, we have

$$
f\left(B_{n} x\right)<f(x)-\delta
$$

for each $n=1,2, \ldots$.

Choose a positive integer $N$ such that

$$
\delta N>d_{B_{0}}-\inf (f),
$$

Now assume that $\left\{B_{n}\right\}_{n=1}^{\infty} \in U$ and $r:\{1,2, \ldots\} \rightarrow\{1,2, \ldots\}$. We claim that

$$
f\left(B_{r(N)} \ldots B_{r(1)} B_{0} x\right)<\inf (f)+\varepsilon
$$

for each $x \in K$. Suppose to the contrary that this is not true. Then there exists a point $x \in K$ such that

$$
f\left(B_{r(n)} \ldots B_{r(1)} B_{0} x\right) \geq \inf (f)+\varepsilon, n=0, \ldots, N .
$$

Using (5.5) and induction, we see that, for each $n=1, \ldots, N$,

$$
f\left(B_{r(n)} \ldots B_{r(1)} B_{0} x\right)<f\left(B_{0} x\right)-n \delta .
$$

This implies that

$$
f\left(B_{r(N)} \ldots B_{r(1)} B_{0} x\right)<f\left(B_{0} x\right)-N \delta<d_{B_{0}}-\left(d_{B_{0}}-\inf (f)\right)=\inf (f),
$$

a contradiction. Therefore, (5.6) is indeed valid, as claimed, and Theorem 5.3 is established.

\section{PRoOfS OF THE MAIN RESUlTS}

Theorem 2.1 is a direct consequence of Theorems 4.1 and 5.1. Theorem 2.2 is a direct consequence of Theorems 4.2 and 5.2. Theorem 2.3 is a direct consequence of Theorems 4.3 and 5.3. 


\section{NORMALITY, WEAK NORMALITY AND METRIC PROJECTIONS ONTO CLOSED BALLS}

Let $d$ be the metric induced on $K$ by $\|\cdot\|$. We denote by $B\left(x_{0}, r\right)$ the closed ball in $(K, d)$ of center $x_{0}$ and radius $r>0$. Since $K$ is convex, there exists a metric projection $P_{B\left(x_{0}, r\right)}$ of $K$ onto $B\left(x_{0}, r\right)$. If $X$ is a strictly convex Banach space, then this metric projection is unique. By taking $B_{0}=P_{B\left(x_{0}, r\right)}$ in our main results we obtain the following corollaries.

Corollary 7.1. There exist sets $\mathscr{F} \subset \mathfrak{M}, \mathscr{F}_{b} \subset \mathscr{F} \cap \mathfrak{M}_{b}, \mathscr{F}_{c} \subset \mathscr{F} \cap \mathfrak{M}_{c}$ and $\mathscr{F}_{b c} \subset \mathscr{F}_{b} \cap \mathfrak{M}_{c}$

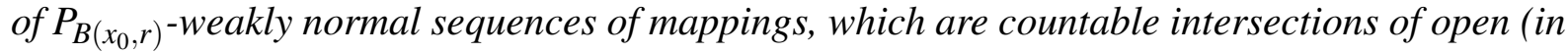
the relative weak topology) and dense (respectively, in the weak topology, in the relative strong topology, in the relative weak topology and in the relative strong topology) sets in, respectively, $\mathfrak{M}, \mathfrak{M}_{b}, \mathfrak{M}_{c}$ and $\mathfrak{M}_{b c}$ such that for each $\left\{A_{n}\right\}_{n=1}^{\infty} \in \mathscr{F}$, the following assertion holds:

For each $\varepsilon>0$, there exist a neighborhood $U$ (in the weak topology) of $\left\{A_{n}\right\}_{n=1}^{\infty}$ and a positive integer $N$ satisfying

$$
f\left(B_{N} \ldots B_{1} x\right)<\inf (f)+\varepsilon
$$

for all $\left\{B_{n}\right\}_{n=1}^{\infty} \in U$ and $x \in B\left(x_{0}, r\right)$.

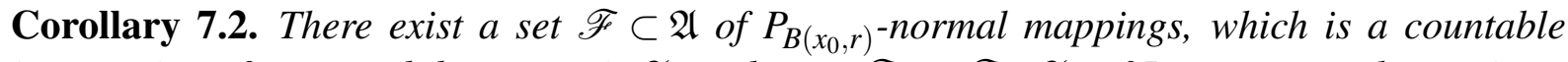
intersection of open and dense sets in $\mathfrak{A}$, and a set $\mathscr{F}_{c} \subset \mathscr{F} \cap \mathfrak{A}_{c}$ of $P_{B\left(x_{0}, r\right)}$-normal mappings, which is a countable intersection of open and dense sets in $\mathfrak{A}_{c}$, such that for each $A \in \mathscr{F}$, the following assertion holds:

For each $\varepsilon>0$, there exist a neighborhood $U$ of $A$ in $\mathfrak{A}$ and a positive integer $N$ satisfying

$$
f\left(B^{N} x\right)<\inf (f)+\varepsilon
$$

for all $B \in U$ and $x \in B\left(x_{0}, r\right)$.

Corollary 7.3. There exist sets $\mathscr{F}_{b} \subset \mathfrak{M}_{b}$ and $\mathscr{F}_{b c} \subset \mathscr{F}_{b} \cap \mathfrak{M}_{c}$ of $P_{B\left(x_{0}, r\right)}$-normal sequences of mappings, which are countable intersections of open (in the relative strong topology) and dense (in the relative strong topology) sets in, respectively, $\mathfrak{M}_{b}$ and $\mathfrak{M}_{b c}$, such that for each $\left\{A_{n}\right\}_{n=1}^{\infty} \in \mathscr{F}$, the following assertion holds:

For each $\varepsilon>0$, there exist a neighborhood $U$ (in the strong topology) of $\left\{A_{n}\right\}_{n=1}^{\infty}$ and a positive integer $N$ satisfying

$$
f\left(B_{r(N)} \ldots B_{r(1)} x\right)<\inf (f)+\varepsilon
$$

for all $\left\{B_{n}\right\}_{n=1}^{\infty} \in U$, all mappings $r:\{1,2, \ldots\} \rightarrow\{1,2, \ldots\}$ and all points $x \in B\left(x_{0}, r\right)$.

\section{Acknowledgments}

The second author was partially supported by the Israel Science Foundation (Grant 820/17), the Fund for the Promotion of Research at the Technion and by the Technion General Research Fund.

\section{REFERENCES}

[1] J. L. Kelley, General Topology, D. Van Nostrand, New York, 1955.

[2] K. Barshad, S. Reich, A. J. Zaslavski, Generic properties of normal mappings, Linear Nonlinear Anal. 6 (2020), 205-218. 
[3] M. Gabour, S. Reich, A. J. Zaslavski, A class of dynamical systems with a convex Lyapunov function, in "Constructive, Experimental, and Nonlinear Analysis", Canadian Mathematical Society Conference Proceedings, Vol. 27, pp. 83-91, 2000.

[4] S. Reich, A. J. Zaslavski, On the minimization of convex functionals, in "Calculus of Variations and Differential Equations", Res. Notes Math. 410, pp. 200-209, Chapman \& Hall/CRC, Boca Raton, Fl, 2000.

[5] M. E. Rudin. A new proof that metric spaces are paracompact, Proc. Amer. Math. Soc. 20 (1969), 603.

[6] E. Michael, Continuous selections. I, Ann. Math. 63 (1956), 361-382.

[7] S. Reich, A. J. Zaslavski, Genericity in Nonlinear Analysis, Springer, New York, 2014. 